\title{
Quejas y expectativas de estudiantes de licenciatura en lenguas extranjeras frente a las clases de inglés: estudio de caso desde el análisis crítico del discurso ${ }^{1}$
}

\author{
Complaints and expectations of students of Bachelor \\ of Arts in Foreign Language regarding English classes: \\ A case study from the perspective of critical discourse \\ analysis
}

\author{
Ruth Elena Quiroz Posada² \\ Ana Elsy Díaz Monsalve ${ }^{3}$
}

Citation/ Para citar este Artículo: Quiroz Posada, R. E., \& Díaz Monsalve, A. E. (2019). Quejas y expectativas de estudiantes de licenciatura en lenguas extranjeras frente a las clases de inglés: estudio de caso desde el análisis crítico del discurso. Colomb. Appl. Linguistic. J., 21(2), pp. 260-275.

Received: 08-Nov.-2018 / Accepted: 15-May.-2019

DOI: https://doi.org/10.14483/22487085.14065

\section{Resumen}

Se exponen los resultados de un proyecto de investigación en el aula como estudio de caso, en el que se indagó a un grupo de estudiantes por situaciones vividas en las clases de inglés. Se identificaron en ellas sus quejas y sus expectativas; se implementó un taller total (Egg, 1999) a fin de reconocer dichas situaciones. El tratamiento dado a la información recogida en las quejas y expectativas de los estudiantes, que consistió en el análisis crítico del discurso (Van Dijk, 2003) y su interpretación desde la pedagogía y la Teoría del Reconocimiento (Honneth, 1997), permitió revelar, más allá de los motivos por los cuales se quejaban, las características de la clase de inglés esperadas por los estudiantes, relacionadas con algunas categorías didácticas y las cualidades de los docentes de esta área conexas con el profesionalismo, la calidez y la interacción. La ausencia o escasez de tales características y cualidades esperadas respectivamente, dificulta el aprendizaje significativo en el inglés al no ofrecer a los estudiantes condiciones justas, indispensables para su proceso formativo de ciudadanos integrales y reconocidos en las dimensiones del amor, el derecho y la solidaridad.

Palabras clave: categorías didácticas, justicia, narrativas, pedagogía, Teoría del Reconocimiento

\begin{abstract}
A classroom-based case study was conducted as part of this research project to understand situations experienced by a group of students during their English classes. The students' complaints and expectations in each context

1 Este artículo es el resultado de un proyecto de investigación en el aula, respondiendo a la modalidad de estudio de caso. Responde a objetivos planteados por el grupo de investigación "Pedagogía y Didáctica de las Lenguas Extranjeras", de la Escuela de Idiomas de la Universidad de Antioquia, específicamente en su línea "Componente pedagógico y didáctico en la formación de licenciados en lenguas extranjeras".

2 Universidad de Antioquia, Medellín (Colombia). ORCID (ID https://orcid.org/0000-0003-2726-8288. ruth.quiroz@udea.edu.co

3 Universidad de Antioquia, Medellín (Colombia). ORCID iD https://orcid.org/000-0003-4017-5182. ana.diaz@udea.edu.co
\end{abstract}


were identified. An overall workshop (Egg, 1999) was introduced in order to recognise such situations. The information collected through students' complaints and expectations was processed on the basis of critical discourse analysis (Van Dijk, 2003), in addition to its interpretations from the perspectives of pedagogy and the theory of recognition (Honneth, 1997), thus, allowing to reveal students' expectations of English classes beyond their complaints. These characteristics are related to educational categories and teachers' qualities as regards professionalism, warmth and interaction. The lack of these expected characteristics and qualities makes meaningful English learning difficult because students are unable to experience fair conditions that are essential for their educational process in order to become comprehensive citizens, recognised in terms of love, law and solidarity.

Keywords: educational categories, justice, narratives, pedagogy, theory of recognition

\section{Introducción}

En este artículo se propone hacer una reflexión acerca de un proyecto de investigación en el aula, cuyo objetivo fue analizar las situaciones más frecuentes que son motivo de queja para un grupo de estudiantes de tercer semestre de un programa de licenciatura en lenguas extranjeras, en una institución pública de educación superior de la ciudad de Medellín. Las quejas giraban alrededor de lo que han vivido en las clases de inglés, específicamente.

Al tomar como fundamento los motivos de estas quejas, se trascendió a identificar aquellas expectativas de la clase de inglés y del docente, que esperan ver los estudiantes. La interpretación de estos motivos se hizo con base en la pedagogía y la teoría del reconocimiento de Axel Honneth (1997). Con esta guía se asumió la consideración teórica según la cual, si la clase o el docente de inglés no reúnen ciertas características o cualidades, se podría estar impidiendo el proceso de aprendizaje significativo y la formación que se les debe ofrecer a los estudiantes para ser ciudadanos integrales, justos y competentes (Orozco, 2002; Orrego y Díaz, 2010; Tobón, 2010).
Entre los docentes y coordinadores de las instituciones educativas son bien conocidas las quejas de los estudiantes, que evidencian su inconformismo con lo que ven en sus docentes y lo que viven en las clases y en la institución educativa. Los aspectos de mayor inconformismo son: las evaluaciones, las metodologías, el exceso de tareas, las actividades remediales, asuntos relacionados con la disciplina, los recursos, etc. Para los estudiantes se trata de situaciones "injustas", por lo cual dicha valoración demanda analizar con mayor profundidad qué hay tras esas expectativas y quejas o inconformismos, las cuales pueden ser situaciones generadoras de conflictos, violencia y agresiones entre docentes y estudiantes.

Indagando estudios realizados alrededor del tema de los conflictos en las aulas de instituciones educativas, se resalta el de Baggini (2012), quien afirmó en su trabajo que era necesaria "una aproximación al análisis del contenido de las representaciones sociales sobre la violencia escolar en una escuela secundaria de la ciudad de México", estudio este que visibiliza el significado social que tienen los actores de la escuela sobre la violencia dada en esta y su concreción en prácticas agresivas, lo cual permite para la presente investigación un primer acercamiento a aquello que puede estar actuando como factor de quejas suscitadas por motivos interpretados por los estudiantes como injustos, los cuales, si no se atienden, pueden detonar en comportamientos violentos. Mateos (2015) explora la categoría de violencia desde la perspectiva de las reflexiones infantiles y adolescentes, para lo cual analiza relatos escritos de niños, niñas y jóvenes que fueron reunidos en un espacio de participación política; integra en su trabajo el enfoque de Bustelo y Lombardo (2007), para interpretar las narrativas como constructoras de ciudadanía juvenil. De otro lado, Ramírez (2014) estudia las identidades juveniles, específicamente ligadas a una narrativa que la autora denomina "caleña", con el fin de solucionar algún conflicto juvenil violento entre jóvenes, para lo cual busca construir tipologías teóricas transdisciplinares y diseñar estrategias de intervención que permitan la transformación positiva del conflicto.

Una manera de prevenir los actos violentos es, justamente, permitir que los estudiantes expresen 
sus inconformidades. Las quejas no atendidas, los malestares no elaborados y los silencios a presión desencadenan manifestaciones agresivas que deterioran las relaciones interpersonales entre quienes habitan la institución educativa (Salm y Gómez, 2000; Ghiso y Ospina, 2010) y, más específicamente, quienes interactúan en el aula de clase. Cuando los estudiantes manifiestan sus quejas lo hacen muchas veces en el tono de quien se siente ofendido. De acuerdo con esto, se ve entonces la necesidad de crear condiciones para que los estudiantes expresen sus desacuerdos y con libertad manifiesten su sentir, de cara a plantear soluciones que disminuyan los niveles de violencia escolar.

De otro lado, las consultas en bases de datos sobre este tema, particularmente entre los estudiantes universitarios que cursan el programa de licenciatura en lenguas extranjeras, no reportaron bibliografía relacionada, ni en general ni en las clases de lengua extranjera (inglés) en particular. Esta realidad motivó a las autoras a plantear la siguiente pregunta de investigación y orientar con ella el proyecto de aula: ¿Cuáles son las situaciones más frecuentes por las que se quejan algunos estudiantes de un programa de licenciatura en lenguas extranjeras de una institución pública de educación superior de la ciudad de Medellín, y cómo se relacionan con lo que han vivido específicamente en las clases de inglés?

Así, con base en las quejas se busca identificar cuáles son las características y cualidades que respectivamente los estudiantes esperan ver en las clases y en los docentes de esta área.

A partir de lo anterior, el objetivo general de la investigación fue analizar las situaciones (motivos) más frecuentes por las que se quejan los estudiantes del tercer semestre del programa Licenciatura en Lenguas extranjeras, y su relación con lo que han vivido específicamente en las clases de inglés, identificando en ellas características $\mathrm{y}$ cualidades que esperan ver respectivamente en las clases y en los docentes de esta área. Y como parte de los objetivos específicos: en primer lugar, se propone un taller a los estudiantes en el que se les problematicen situaciones vividas en las clases de inglés; en segundo lugar, se trata de reconocer los motivos de las quejas de los estudiantes vividas en la clase de inglés en la universidad en el programa de la Licenciatura en Lenguas extranjeras haciendo un recorrido desde su experiencia en el colegio; y, en tercer lugar, se busca identificar en el contenido de las quejas las posibles características y cualidades que los estudiantes de inglés esperan ver en las clases y en los docentes de esta área. Esta indagación se hace fundamentada en la pedagogía y la Teoría del Reconocimiento.

\section{Marco conceptual}

\section{La formación como objeto de la pedagogía y su apoyo en la ley educativa}

La pedagogía tiene como objeto fundamental de reflexión teórica y metodológica el proceso de formación a través del cual se orienta el desarrollo de la personalidad de los estudiantes hacia el logro de unos fines determinados, en coherencia con lo que demanda el contexto social en un momento histórico concreto.

La formación de un ciudadano integral (Orozco, 2002; Tobón, 2010) es, pedagógicamente hablando, la meta del sistema educativo colombiano (Constitución Política de 1991; Ley 115 de 1994, Artículo 5º ), a ello se llega: a) Con la implementación de didácticas que favorezcan la posibilidad que los estudiantes desplieguen sus potencialidades humanas -físicas, espirituales, psicológicas, cognitivas, emocionales y colectivas-, en los procesos de enseñanza y aprendizaje, orientados a actividades, espacios y recursos que implican la planeación de acciones educativas, instructivas y desarrolladoras (Díaz y Quiroz, 2005), acordes con las características personológicas de los estudiantes, con las particularidades del contexto social y cultural, y con la naturaleza del saber específico que el docente orienta para su aprendizaje, en este caso el inglés; b) Con cualidades de los docentes que favorecen los procesos de aprendizaje significativo de los estudiantes en las diferentes áreas y aportan al proceso de formación integral de los ciudadanos. 
Desde este punto de vista, es necesario hacer un esfuerzo por analizar qué pretende el Estado colombiano cuando hace referencia a la formación de un ciudadano integral y cuál es el contenido que le da a dicha educación. De acuerdo con el Artículo 16 de la Constitución Política Nacional, los estudiantes tienen derecho "al libre desarrollo de su personalidad sin más limitaciones que las que imponen los derechos de los demás y el orden jurídico". La educación es el medio para que se haga efectivo el cumplimiento de este derecho, a medida que, en ese proceso de formación, el estudiante construye conocimientos y desarrolla habilidades, actitudes y valores que lo posicionan a la altura de su época. Y para que esto se logre se deben garantizar todas las condiciones institucionales, de la clase y de los maestros.

El Artículo 67 de la Constitución Política y, más precisamente, la Ley General de Educación de 1994 en su artículo 5º precisan lo que debe contemplar la formación de ciudadanos integrales y para ello exponen los fines de la educación colombiana. En este marco, la educación busca "el pleno desarrollo de la personalidad [...] dentro de un proceso de formación integral, física, psíquica, intelectual, moral, espiritual, social, afectiva, ética, cívica y demás valores humanos". Este propósito se encuentra en la base de los demás propósitos que contempla la Ley de Educación. Por otro lado, el Artículo 68 de la Constitución incluye que para el logro de lo anterior: "La enseñanza estará a cargo de personas de reconocida idoneidad ética y pedagógica", que reconozcan el derecho que asiste a los estudiantes de formar parte de procesos de formación, de enseñanza y de aprendizaje justos.

Por esta vía de argumentación, se infiere que la escuela debe ofrecer herramientas para que los estudiantes adquieran una formación ciudadana (Congreso de Colombia, Ley 115 de 1994), entendida como un proceso permanente de aprendizaje que no está agenciado ni determinado por una institución particular, sino que está conformado por una trama de aprendizajes que se dan en todos los espacios de aparición (entre los que se incluye la institución escolar) y escapan a procesos de objetivación. En ellos, se critican los efectos causados específicamente en las denominadas competencias ciudadanas (Benjumea, Gutiérrez, Jaramillo, Mesa y Pimienta, 2011). Además, se entiende que es un deber de la escuela, contar con un personal docente que reúna las cualidades necesarias de idoneidad ética y pedagógica para proporcionar este servicio educativo (Constitución Política de Colombia, 1991).

Por eso, teniendo en cuenta el objetivo de este estudio, cuando un estudiante manifiesta su sentir acerca de sus experiencias, expectativas o situaciones, hace un gran aporte que ayudaría a reconocer qué está fallando desde el punto de vista pedagógico en cuanto a la educación que se le ofrece y la formación que se le orienta, así como las cualidades que la clase y el docente deben reunir para hacer que este proceso de formación se aproxime, al menos, a lo que se ha postulado en la ley. No permitir la queja implica lesionar los derechos a recibir una formación integral de parte de un agente competente para ello: la queja, así, es un medio de denuncia que contribuye a impedir que se sigan vulnerando los derechos y que se continúen reproduciendo estas situaciones (Mateos, 2015). Si al estudiante se le permiten espacios para que dé a conocer sus inconformidades, se contribuye a disminuir conflictos y posibles actos de violencia en el aula y se aclara el camino a seguir en tanto se plantean acciones que transformen positivamente la situación en cuestión. Este proceso evita que se complejicen los procesos o se convierta en un problema mayor para los actores o para la institución.

En el contexto específico de la clase, si lo planteado en la ley responde al ideal de formación pedagógica, la didáctica con la que se desarrolla la clase en general y la de la lengua extranjera en particular, se debe estructurar desde sistemas didácticos que integren las categorías de problemas de enseñanza y de aprendizaje, objetivos de la clase, contenidos por desarrollar, métodos que se deben implementar, formas de organización de la clase, recursos de apoyo a la didáctica y evaluación de los aprendizajes y las competencias (Álvarez y González, 1998; Díazy Quiroz, 2005), en medio de dinámicas de interacción y comunicación que permitan relaciones de cercanía, respeto, cuidado y consideraciones necesarias entre docentes y estudiantes. 
Finalmente, en lo que tiene que ver con las cualidades propias del maestro, en coherencia con la ley, se espera que este posea ciertas características —profesionalismo, calidez, interacción-, para el ejercicio idóneo y profesional de su labor. Este marco permitiría ver hasta qué punto se está siendo justo o injusto con el ofrecimiento de una educación planteada por el Estado y requerida por el estudiante.

\section{La justicia educativa en la teoría del reconocimiento de Axel Honneth}

Es un asunto de justicia ofrecer a los estudiantes una educación que les forme de manera integral, poniendo al servicio de este fin todo lo que tiene que ver con el desarrollo de las clases y la actuación misma del maestro en ellas. En relación con esto, el análisis pedagógico de las quejas de los estudiantes desde la teoría del reconocimiento de Honneth (1997) permite ver que, en el fondo, lo planteado en la Ley General de Educación (2012) reclama la necesaria formación del estudiante, ciudadano concreto, en la autoconfianza, el autorrespeto y la autoestima (Honneth, 1997, citado por Mejía, 2017), con fundamento en la implementación de modos de reconocimiento en la clase que faciliten por parte del docente una dedicación emocional, una atención cognitiva y una valoración social de los estudiantes: en esto vemos las cualidades de los docentes esperadas por los estudiantes.

El docente debe diseñar su didáctica de tal forma que en las clases se haga evidente cómo orientará el desarrollo de las dimensiones en los estudiantes esferas del reconocimiento según Honneth-: amor, derecho y valoración social (solidaridad), atendiendo a las necesidades que puedan tener en cada una de ellas, con el propósito de lograr la meta pedagógica proyectada en las autorrealizaciones mencionadas -autoimagen, autoestima, autoconfianza, autorrespeto y autocuidado- reflejadas en la ley. Frente a ello se procede a hacer algunas claridades:

Primero, el amor desde la teoría del reconocimiento de Axel Honneth, implica una dedicación emocional que favorece la autoconfianza, gracias a la cual se mejoran las interrelaciones entre las personas de manera espontánea y natural. En el plano de la educación, según esta teoría, las relaciones de amistad y de afecto favorecen una formación en la autoimagen y la autoestima de las propias capacidades personales integralmente vistas -afectivas, cognitivas, sociales, individuales - que facultan al estudiante para una participación autónoma, activa y eficiente en el contexto social.

Hacer una lectura de esta esfera del reconocimiento en el plano de lo educativo, lo pedagógico y lo didáctico dado en la institución educativa y en el aula de clase, permite inferir la importancia de la relación cálida y acogedora entre docente y estudiante como respuesta a la necesidad sentida por este de que su profesor esté pendiente de él, en cuanto a lo que tiene que ver con su proceso de formación integral (Orozco, 2002), sus avances en el desarrollo cognitivo y emocional, sus aprendizajes, sus potencialidades y sus dificultades para aprender, y además la preocupación por cómo avanza su estudiante para brindarle posibilidades en cuanto a estrategias de aprendizaje variadas (Orrego y Díaz, 2010) u otras formas alternativas que apoyen el proceso. El comportamiento autónomo del estudiante sería el fruto de una dimensión afectiva bien desarrollada, que le represente comportamientos de independencia cognitiva y de reconocimiento de su estilo para aprender, así como de sus dificultades y de lo que puede hacer para superarlas.

Segundo, el derecho para la teoría del reconocimiento implica una atención cognitiva que favorece el autorrespeto, indispensable en la formación para la convivencia regulada por la voluntad colectiva que, representada en la norma, permite ambientes de paz y la solución dialogada de los desacuerdos y los conflictos. Hacer una lectura de esta esfera del reconocimiento en el plano pedagógico y didáctico que se da en la institución educativa y en el aula de clase, permite inferir la importancia de favorecer situaciones formativas en las que se enfaticen la participación y la dinámica colectiva $-\mathrm{y}$ no sólo individual- por parte de los estudiantes, por medio de estrategias que permitan la discusión respetuosa, el diálogo, la elaboración de consensos, disensos y acuerdos intersubjetivos y la voluntad de respeto y consideración hacia todos y cada uno 
de los compañeros de clase y demás personas que trabajan en la institución. Respetar el derecho propio conduce a la consciencia del respeto hacia los otros. Lo contrario, no permitirle al estudiante estas posibilidades, conduce a exclusiones, desigualdades e invisibilizaciones que podrían motivar sentimientos, pensamientos y acciones de subvaloración, un hecho que deteriora el autorrespeto.

Tercero, la dimensión de la solidaridad para la teoría del reconocimiento implica la valoración social que favorece el autocuidado y la autoconfianza manifiestas en acciones relacionadas con la cooperación, con el compartir y valorar lo que el otro puede ofrecer. Sentirse apoyado y sostenido por la solidaridad del profesor o de sus compañeros, en el caso de los estudiantes, favorece la formación en el autocuidado y la autoconfianza que les faculta para dar todo el potencial de sí, puesto desde los andamiajes que se brindan. En el plano de la educación, las relaciones de solidaridad y de cooperación se facilitan con la valoración social que se le hace al estudiante por sus conocimientos, procedimientos y actitudes así como al ofrecerle andamiajes precisos y oportunos para superar los vacíos, las limitaciones y dificultades que presentan. Gozar de valoración social y de consideración en medio del grupo de estudiantes es algo que el docente puede motivar, lo cual facilita la participación en los procesos individuales de aprendizaje. Si no se favorece esta dimensión de la solidaridad, con facilidad se incurrirá en acciones, según Honneth (1997), de injuria e indignidad, que frenan la realización personal o colectiva.

Como se puede apreciar en las líneas anteriores, una lectura pedagógica de la teoría de Honneth (1997) permite inferir que es cuestión de justicia, desde el reconocimiento, garantizar al educando, en sus clases, condiciones pedagógicas y didácticas que favorezcan el desarrollo de estas dimensiones. También es cuestión de justicia contar con docentes sensibles a estas necesidades formativas del estudiante relacionadas con el amor, el derecho y la solidaridad, y deben poseer las cualidades necesarias como docentes para favorecerlas y potenciarlas. Caben las preguntas: ¿se está cumpliendo con ello?, ċcuál es la principal queja de los estudiantes en su paso por la enseñanza del inglés en el colegio o la universidad?

\section{Metodología}

Este proyecto de aula se llevó a cabo con 29 estudiantes de un programa de Licenciatura en lenguas extranjeras, matriculados en uno de los cursos de formación pedagógica que conforman el tercer semestre del plan de estudios. Los estudiantes tienen las siguientes características: diecisiete hombres y doce mujeres; sus edades oscilan entre diecinueve y veinticuatro años; y son de estrato socioeconómico uno, dos y tres.

El proyecto de investigación se orientó a partir del paradigma cualitativo con un enfoque interpretativo que permitió generar, como afirma Galeano (2004, p. 15), "un modo de encarar el mundo de la interioridad de los sujetos sociales y de las relaciones que establecen con los contextos y con otros actores sociales". En este caso interesaba analizar, desde los mismos estudiantes, las situaciones (motivos) más frecuentes por las que se quejan relacionadas con sus vivencias y expectativas, específicamente en las clases de inglés, de cara a identificar qué características esperan ver en las clases y las cualidades que esperan en los docentes de esta área encaminadas a favorecer el amor, el reconocimiento de sus derechos y la solidaridad.

Por esta vía, el estudio de caso utilizó como estrategia de recolección de datos el taller total denominado “¿De qué te quejas"?", en el que se les invitaba a recordar su época de estudio en la escuela y el colegio, y el transcurso en la universidad, a fin de evocar situaciones de clase de las cuales tenían alguna queja. En este sentido, se les orientó hacia la elaboración de unas narrativas en las que debían describir con detalle el motivo de su disgusto y de su queja, y argumentar el porqué de ellas. Las situaciones de queja fueron socializadas en el grupo para permitir mayor participación del grupo en general. Las descripciones que generaron los estudiantes conformaron un conjunto de narrativas

$4 \mathrm{La}$ inquietud por conocer las quejas de los estudiantes, surge justamente, de la necesidad de reconocer desde ellos mismos, las situaciones dadas en el proceso de la clase o en el comportamiento del profesor, que son motivos de su inconformismo y que deben ser consideradas para su corrección con fundamento en una lectura pedagógica y también desde lo que se considera justo/injusto en la teoría del reconocimiento aplicado a la educación. 
que, con su consentimiento informado, se trascribieron y retroalimentaron con ellos mismos para facilitar el proceso de análisis.

Por la forma narrativa en la que se presentaron las quejas de los estudiantes, se consideró necesario, en un primer momento, reconocer en ellas información fundamental para responder a la pregunta de investigación. Para ello, se utilizó la técnica del análisis crítico del discurso de Van Dijk (2003), y de manera análoga a como esta técnica fue utilizada por Echavarría (2011, pp. 203-204), con base en los siguientes pasos:

Análisis de las macroestructuras semánticas: hace referencia a los temas - macroproposicionesplanteados con coherencia y cohesión discursiva en la narrativa y específicamente en los testimonios seleccionados (en este estudio son los temas fundamentales o núcleos temáticos desarrollados en las narrativas y testimonios y que respondían con coherencia a la pregunta que motivó la investigación);

Análisis de significados locales: hace referencia a aspectos como implicaciones, contrastes, presuposiciones, alusiones, ambigüedades, omisiones que se detectan en las narrativas y en los testimonios seleccionados (en este estudio son las interpretaciones e inferencias de este tipo desde la pedagogía y la teoría del reconocimiento evidenciado en los testimonios);

Análisis de las estructuras formales: hace referencia a énfasis, coherencias, estructuras proposicionales identificadas en las narrativas y en los testimonios seleccionados (en este estudio las estructuras formales son aquellas categorías de análisis que integran los temas o núcleos temáticos identificados de manera reiterativa en los discursos de los testimonios, relacionados, de una parte, con las cualidades que esperaban ver en las clases de inglés y, de otra parte, con las cualidades que esperaban ver en el docente de esta área);

Análisis de las formas y formatos del discurso global y local: hace referencia al estilo que asume el testimonio seleccionado entre narrativo, descriptivo, explicativo, argumentativo (en este estudio es la caracterización que se le reconoce a la forma en que se presenta el testimonio, desde los tipos de las narrativas mencionadas);

Análisis de realizaciones lingüísticas específicas: hace referencia al uso de recursos literarios o figuras retóricas como las hipérboles y las metonimias que se reconocen en la narrativa y los testimonios seleccionados. (En este estudio es el uso de recursos literarios reconocidos para narrar, describir, explicar o argumentar una situación expuesta en el testimonio). ${ }^{5}$

En general, seguir este procedimiento de análisis en cada uno de los pasos anotados arrojó información abundante que se filtró, sin perder información esencial. Este proceso se hizo mediante lectura y relectura de las narrativas; identificación de frases y expresiones de testimonios fundamentales; reducción de esos testimonios extrayendo las partes con más sentido para efectos de la presente investigación. Posteriormente, se hizo una identificación de los núcleos temáticos emergentes en el conjunto de los testimonios, se hizo una selección y clasificación de testimonios y finalmente se identificaron relaciones entre ellos. En un segundo momento, y ya reducido el volumen de la información, se realizó una interpretación de la información más significativa desde la pedagogía y la teoría del reconocimiento.

Las quejas puestas en evidencia en las narrativas se analizaron desde la teoría pedagógica y la teoría del reconocimiento de Honneth (1997) en sus dimensiones del amor, el derecho y la solidaridad, en aras de hallar, desde lo compartido en estas narrativas, las áreas que los estudiantes calificaban como más afectadas, para que mediante estas se pudiera tener una visión sobre lo necesario para ofrecer una educación justa que se solidariza con el logro de los fines de la educación planteados en la Ley General de Educación.

\section{Análisis crítico del discurso}

Al analizar el estilo de los discursos narrativos de los estudiantes que participaron en la investigación

5 Lo que se presenta en letra cursiva corresponde a la denominación original de cada paso anotado según su autor Van Dijk (2003). 
se evidenció que prevalecen el descriptivo y el argumentativo, en ocasiones el estudiante describe situaciones sobre lo que se le pregunta, en otras hace breves narraciones en las que ofrece características en cuanto a lo que está respondiendo y en otras trasciende al ofrecimiento de argumentaciones para ampliar su punto de vista. Los núcleos temáticos que se pudieron construir alrededor de las quejas realizadas por los estudiantes relacionadas con las clases y los docentes de inglés son los siguientes: el primer núcleo temático centrado en las quejas sobre las clases de inglés; el segundo núcleo temático centrado en las quejas sobre los docentes. El análisis trascendió el motivo de la queja hacia identificar las características de la clase y las cualidades del docente que los estudiantes esperan ver en la enseñanza del inglés.

Primer núcleo temático: quejas sobre las clases de inglés

Este primer núcleo temático integra testimonios que aluden a las características que presenta la clase de inglés, con especialidad a las categorías didácticas de los contenidos, los métodos de enseñanza, los recursos de apoyo a la didáctica y la evaluación (Díaz y Quiroz, 2005). Aunque en sus quejas los estudiantes describen las características de la clase de inglés con las cuales se sienten inconformes, estas mismas características nos orientan entonces sobre cuáles son sus expectativas.

\section{Quejas sobre los contenidos de la clase de inglés}

En este aspecto las quejas giran alrededor de clases en las que, según los estudiantes que participaron, se les transmite mucha información, pero no se elabora desde el punto de vista cognitivo, de tal manera que no se favorece un aprendizaje significativo. O clases en las que el contenido enseñado era no solo "innecesario", sino "obsoleto" (Estudiante 21):

\footnotetext{
"[Me quejo] de tantos profesores que [...] no enseñaban NADA. No servía para nada [...]". (Estudiante 4)

"Me quejo de algunos docentes de inglés por la cantidad de información innecesaria, temas de clase obsoletos". (Estudiante 21)
}

Con base en el contenido de estos testimonios, se infiere que el estudiante espera que en las clases de inglés se traten y estudien contenidos significativos, no necesariamente abundantes, pero sí analizados con profundidad, contenidos actuales y cuya utilidad se pueda reconocer, con objetivos para cada clase y con transferencia a situaciones de la vida práctica. El estudiante reclama la enseñanza de contenidos pertinentes y contextuales, que respondan a sus necesidades y a las del contexto en el que habita; contenidos de la ciencia, para que pueda participar en la sociedad con unos conocimientos confiables, válidos y pertinentes. No es la cantidad de información lo que el estudiante solicita desde la dimensión del derecho, sino la calidad de los contenidos de lo que se enseña y la elaboración cognitiva que se hace con ellos, lo cual favorece aprendizajes significativos. Los responsables de confirmar que así sea son la sociedad, el Estado y la familia.

\section{Quejas sobre los métodos de la clase de inglés y los recursos utilizados en ella}

Las quejas relacionadas con el método de enseñanza muestran que las clases se caracterizan por ser "improvisadas" y "monótonas" para enseñar los diferentes contenidos. Acá sería muy importante apelar a dinamizar estos métodos con el uso de estrategias de aprendizaje propias para la enseñanza de la lengua extranjera (Orrego y Díaz, 2010). Sobre este caso los estudiantes expresan:

\footnotetext{
"Me quejaría de las clases improvisadas sin ninguna planeación". (Estudiante 4)

"[...] de las clases monótonas y que el profesor solo tenga un método de enseñanza, cuando cada persona aprende de una forma y [a] un ritmo diferentes". (Estudiante 27)

"Me quejaría de tantas clases cuadriculadas". (Estudiante 29)
}

Dicha monotonía la favorece la implementación de un único método de enseñanza, que no varía ni por razón del contenido ni de los estilos cognitivos o los ritmos de aprendizaje de los estudiantes. Monotonía y carácter de "cuadriculado" que se llegan a explicar en la rutina metodológica en la que se acomoda el docente; monotonía por la escasa variación en las estrategias de enseñanza y el uso de materiales 
didácticos que no llaman la atención y no permiten una aproximación al tema de clase de manera directa. En términos pedagógicos, se pone en evidencia la existencia de clases que responden a un modelo pedagógico tradicional (Díaz y Quiroz, 2005). Esto lo observan los estudiantes, quienes sintetizan que "no tienen una pedagogía para enseñar" (Estudiante 1) y que les faltan "herramientas pedagógicas" (Estudiante 11). Y en estas condiciones solo les queda "enseñar siempre de la misma manera, con el mismo material" (Estudiante 17). Los siguientes testimonios sintetizan en gran medida la queja de los estudiantes en el aspecto tanto de la metodología como de los materiales de apoyo didáctico presentados por los docentes:

\begin{abstract}
"[Me quejo de] Maestros que dan una clase en la que se sientan en su escritorio a repartir fotocopias y poner consultas, las cuales califican 'mientras más hojas mejor', y si les haces una pregunta responden: 'eso está en la consulta". (Estudiante 8)

"En ocasiones se notaba la poca preparación de las clases. Y [los docentes] hacían ciertas actividades para hacer tiempo, pues no estaban enfocadas a los objetivos de las clases. Asimismo, la metodología que utilizaban para dictar sus clases casi siempre era pasar de un libro al cuaderno". (Estudiante 26)
\end{abstract}

Se infiere de estos testimonios que los estudiantes esperan ver en las clases de inglés características metodológicas que promuevan en ellos la participación activa, física y cognitiva alrededor de los temas que se tratan; una clase donde se les permita ejercer procesos de pensamiento de orden superior sobre lo que están estudiando y que en un ambiente de diálogo respetuoso puedan aportar con seguridad sus ideas y puntos de vista. Un método así ha de apoyarse en conversaciones y recursos didácticos novedosos, pertinentes y actuales que les llamen la atención y les faciliten una aproximación a aquello que estudian.

\section{Quejas sobre la evaluación implementada en la clase de inglés}

Algunos estudiantes se quejan respecto de las evaluaciones, sobre todo las que no son planeadas, las que solo miden reproducción de conocimientos y no comprensión, y de la calificación que se les da a estas, sin claridad en los criterios de evaluación. He aquí algunos testimonios:

"Me quejo de docentes que evalúan cosas que no enseñan y que no dijeron que iban a evaluar". (Estudiante 1)

"[...] por evaluar solo resultados y no procesos, deberían ser ambos". (Estudiante 3)

"[...] de los criterios evaluativos, pues no están bien organizados y parecen improvisados" (Estudiante 22)

El estudiante encuentra motivos para quejarse de las evaluaciones, cuando se evalúan temas que no fueron tratados en clase, o que no se hizo la claridad que serían objeto de evaluación; cuando en su evaluación solo importa el qué de lo aprendido y no el cómo, ni el para qué y por qué; sobre todo, el estudiante se queja cuando se evalúa sin criterios claros, o no se los dan a conocer, o cuando estos criterios parecen espontáneos, según el estudiante que sea evaluado.

Una evaluación que presente estas características que denuncian los estudiantes en sus quejas, justifica plenamente que se convierta en una "exigencia justa" (Estudiante 4). Por otra parte, también se manifiestan quejas en cuanto a las evaluaciones subjetivas:

"[...] de las calificaciones desordenadas, subjetivas e injustas". (Estudiante 23)

"[...] que el método de calificación siempre es algo muy subjetivo, dependiendo de lo que el profesor piensa que merece cada persona, y sin fijarse muchas veces en los desarrollos, los esfuerzos o en las aptitudes que se muestran". (Estudiante 27)

Es importante anotar que para el estudiante una evaluación injusta puede significar que depende del estado de ánimo del que evalúa.

Segundo núcleo temático: quejas sobre los docentes de inglés

Este segundo núcleo temático integra testimonios de los estudiantes que aluden a las 
Tabla 1. Síntesis primer núcleo temático.

\begin{tabular}{|c|l|}
\hline \multicolumn{1}{|c|}{ Queja } & \multicolumn{1}{c|}{ Expectativa } \\
\hline Sobre el contenido & $\begin{array}{l}\text { Que sean pertinentes, actuales, con fundamento en la ciencia. } \\
\text { Que respondan a sus inquietudes y a las necesidades del } \\
\text { contexto. } \\
\text { Que sean potencialmente transferibles a diferentes contextos. } \\
\text { Que sean en cuanto a la cantidad, mesurados pero trabajados } \\
\text { a profundidad. }\end{array}$ \\
\hline Sobre el método y los recursos & $\begin{array}{l}\text { Que sean activos, lúdicos y permitan la participación de todos } \\
\text { en un marco de respeto y amistad. } \\
\text { Que, a través de diferentes actividades, le ayuden al estudiante } \\
\text { en el proceso de elaboración cognitiva de los contenidos a } \\
\text { tratar de manera progresiva y natural. } \\
\text { Que las actividades integren el uso de recursos didácticos en } \\
\text { buen estado, novedosos, pertinentes con el tema y llamativos. }\end{array}$ \\
\hline Sobre la evaluación & $\begin{array}{l}\text { Que sean programadas con suficiente tiempo. } \\
\text { Que sean objetivas definiendo desde antes los criterios de } \\
\text { evaluación. } \\
\text { Que requieran del estudiante conciencia sobre el contenido, el } \\
\text { proceso, el porqué y el para qué de lo evaluado. }\end{array}$ \\
\hline
\end{tabular}

Fuente: elaboración propia.

cualidades que presentan algunos docentes de inglés que les incomodan y por las cuales se quejan. De igual manera, el contenido de estas quejas nos orienta para identificar las cualidades que los estudiantes esperan ver de sus profesores en esta área.

Quejas por la falta de profesionalismo de algunos docentes: un llamado a la atención cognitiva.

Para los estudiantes, el docente incurre en falta de profesionalismo cuando no manifiesta gusto por su materia o cuando no sabe lo que enseña, pues en ocasiones tiene dentro de su asignación académica la enseñanza de asignaturas para las que no está preparado con un conocimiento riguroso.

De acuerdo con las narraciones ubicadas en este núcleo, el docente falta al carácter de "profesional" (Estudiante 20) cuando muestra pereza para realizar su trabajo, cuando carece de buena voluntad para hacer dinámicas interesantes en sus clases (Estudiante 21), y cuando no apela a toda su capacidad creativa para orientar el aprendizaje de los estudiantes, con lo cual sus clases y el aprendizaje se vuelven monótonos:

\section{"[Me quejaría] de algunos profesores que simplemente no les importa enseñar". (Estudiante 10) \\ "Yo me quejaría de la falta de profesionalismo de ciertos docentes, quienes no están interesados en su materia o en el conocimiento del mundo". (Estudiante 20) \\ "[Me quejaría] de la falta de [...] voluntad por educar [...] y una frustración manifiesta hacia la labor a realizar en la enseñanza". (Estudiante 21)}

Una lectura de estas quejas desde la teoría del reconocimiento (Honneth, 1997) permite identificar 
la dimensión del derecho. Los estudiantes reclaman el derecho que tienen a contar con docentes de inglés formados profesionalmente, que sepan cómo favorecerles en la construcción, utilidad, importancia y pasión por el conocimiento de la materia a su cargo, por medio del diseño y el uso de estrategias didácticas y de acciones pedagógicas justas y necesarias para su proceso de formación, y así cumplir con el derecho a una educación de calidad.

"[...] Algunos no son docentes por gusto sino por motivos meramente monetarios" (Estudiante 28). Esto lo afirma un estudiante en su testimonio; sin embargo, la mayoría de estudiantes relacionan la falta de profesionalismo con lo que denominan "mediocridad". En los testimonios se puede evidenciar que el estudiante primero emplea un estilo descriptivo, presentando las características de lo que para él es un "profesor mediocre", como aquel que no prepara la clase (Estudiantes 11 y 19) y exige poco al estudiante y se conforma con pocos esfuerzos (Estudiante 19), y luego utiliza un estilo argumentativo en su respuesta, para dar las razones con las cuales justifica y aclara su punto de vista:

\footnotetext{
"[Me quejo] de los profes mediocres que no preparan [...] y simplemente exigen poco, porque a ellos no les interesa que uno aprenda, solo cumplen con horas laborales y esperan un sueldo". (Estudiante 19)

"[Me quejo] de muchos profesores que no hacen ni el mínimo esfuerzo por preparar un curso bien y uno se da cuenta de ello". (Estudiante 11) "[Me quejo] de profesores que podrían llamarse 'mediocres', ya fuera porque la misma institución no les permite salirse de los parámetros establecidos o porque los profesores no quieren salir de la zona de confort [...]". (Estudiante 25)
}

De lo anterior se infiere que los estudiantes esperan de sus docentes cualidades pedagógicas y didácticas que les permitan preparar bien las clases; les gustaría que sus docentes fueran personas sensibles y comprometidas con el progreso social que los estudiantes manifiesten en su proceso de formación; que estén atentos a sus necesidades, inquietudes y motivaciones, y que les dediquen tiempo para escucharlos, lo cual no hacen con frecuencia por estar más preocupados en hacer otros trabajos, "reuniones, diligenciar planillas, dictar clase, notas laborales" (Estudiante 19).

Por los testimonios se descubre que el estudiante juzga como mediocre el actuar del docente que no prepare bien sus clases, con lo cual se infiere que improvisa lo que hace, que su "comodidad" (Estudiante 25) le puede llevar a hacer lo mismo siempre y su metodología se convierte en una rutina para él y sus estudiantes.

Cuando se interpretan las situaciones anteriores desde la Teoría del Reconocimiento, se podría decir que desde la mirada del estudiante este comportamiento poco profesional impide el reconocimiento que él demanda en su dimensión del amor, un profesor que se esmere, que se esfuerce, que dedique más tiempo a los estudiantes que a diligenciar formatos para entregar a sus jefes inmediatos. Se requiere un docente que se sienta motivado porque se encuentra con los estudiantes, por enseñarles lo que sabe, lo que a su vez se refleja en clases interesantes, reflexivas y atrayentes.

Cuando un estudiante manifiesta esta queja realmente está denunciando que no se tienen en cuenta sus necesidades por aprender de manera reflexiva y crítica, que las inquietudes que pueda tener respecto a lo que le enseñan no se consideran; reclama su necesidad de formarse y para ello requiere que existan ciertas cualidades en el docente y en la clase de inglés, y en la dinámica con la que se desarrolla el proceso de enseñanza que es responsabilidad del docente; no preparar la clase es evidencia de una desconsideración a su necesidad de formación.

De otro lado, las narraciones permiten interpretar que esta "no preparación de la clase" puede deberse a la falta de interés del profesor, falta de mayor preparación del docente o a una actitud de comodidad, a "la falta de una voluntad por educar [...] y una frustración personológica manifiesta hacia la labor a realizar" (Estudiante 21). Hay una conexión directa en el testimonio del estudiante entre recibir una clase bien preparada y aprender el tema de la clase. El estudiante puede sentirse no reconocido en su dimensión de la solidaridad al no 
recibir unas clases agradables en las que aprenda y se autoexija, en las que el profesor potencie su actitud de entrega, en la preparación de las clases, en la variedad del material de apoyo que utiliza y en el uso adecuado del tiempo.

Quejas sobre algunos docentes de inglés por su comportamiento poco cálido: un llamado a la dedicación emocional.

Este aspecto está relacionado directamente con el aspecto afectivo y su impacto en el aprendizaje:

\begin{abstract}
"[Me quejaría] del mal genio de los docentes, algunos no son docentes por gusto sino por motivos meramente monetarios". (Estudiante 28) "Me siento inconforme con algunos profesores que no tienen calidad humana y no les importan nuestros problemas". (Estudiante 6)

"[Me quejaría de profesores que] no son lo suficientemente tolerantes, al momento de dictar una clase, cuando se les hacen preguntas o cuando al calificar miran solo el resultado y no el proceso". (Estudiante 7)
\end{abstract}

Calidad humana y respeto son manifestaciones afectivas relacionadas con la necesidad del estudiante de ser reconocido en las acciones pedagógicas y didácticas en su dimensión del amor (Honneth, 1997). ¿Qué ven en el comportamiento de algunos docentes para reclamar esto? Algunos docentes de inglés llegan a ser docentes por circunstancias diferentes a la vocación profesional de enseñar, y muestran al estudiante con su comportamiento que su interés está en otro lugar, en otra carrera o profesión. Una queja como esta pone al docente frente a la necesidad de amar lo que está haciendo, el enseñar, y de ejercer la docencia con gran dedicación, de encontrar en esta profesión una manera de ser feliz, ayudándose a sí mismo y a los otros.

Quejas sobre algunos docentes de inglés que no interactúan con ellos: un llamado a la valoración social y a la interacción.

Los estudiantes evidencian su inconformidad con profesores que "no tienen calidad humana" (Estudiante 6), y con ello se hace alusión a una cualidad del docente que se caracteriza por brindar un trato poco amable, por la actitud intolerante y poco paciente o por la falta de interés en las situaciones que viven. Este reclamo se puede fundamentar en que muchos de los estudiantes provienen de hogares poco funcionales y buscan resolver algunas necesidades de afecto en el colegio, en la universidad, con sus compañeros de estudio o con sus profesores, a quienes en muchas ocasiones admiran. Y es asunto de la dimensión del derecho ofrecerle en su estadía por la institución educativa todo aquello que abarca el concepto de "calidad humana".

Garantizar condiciones que movilicen la parte emocional del estudiante tiene que ver con que el docente le muestre afecto, consideración y respeto en el aula de clase, todo lo cual está en consonancia con lo que sugieren algunas de las teorías más actuales de la reestructuración del aprendizaje. La emoción es una variable que puede explicar los aprendizajes y el desarrollo cognitivo, lo cual se concreta en la didáctica crítica (Rojas, 2009) por medio de estrategias que se fundamenten en el diálogo, en las reflexiones grupales, en la negociación de significados, en las argumentaciones, los debates y en las interpretaciones que se hacen de la clase, del contexto y de los mismos estudiantes. Estas estrategias de intervención garantizan un ambiente cálido en el aula, unas relaciones armónicas entre profesoresy estudiantes, lo cual podría contribuir a la seguridad personal en la confianza para interactuar con otros y a resignificar los conocimientos propios con los aportes de los compañeros de clase, bajo la orientación y asesoría permanentes del docente. En general, los estudiantes reclaman docentes que favorezcan ambientes de aprendizaje en los que sientan seguridad y gusto por participar.

Afirman que algunos profesores no se acercan a interactuar con ellos (Estudiante 3), lo que puede indicar que la interacción docente-estudiante se limita a la que se da en el salón de clase, por motivos exclusivamente académicos, y no se extiende a otros espacios educativos que, si son aprovechados, permitirían más cercanía, confianza y optimización del conocimiento de la realidad personal y familiar de los estudiantes, de sus situaciones y de cómo se les podría ayudar: 
"[Me quejo] de la falta de [...] interacción de muchos de mis profesores hacia el cuerpo estudiantil". (Estudiante 3)

"[Me quejo] de aquellos profesores sin dinámica e interacción en el aula entre ellos y los estudiantes". (Estudiante 3)

Espacios y momentos diferentes al de la clase propiamente dicha podrían ser útiles para posibilitar estas cercanías: el descanso, la cafetería, el corredor, la plazoleta, la biblioteca, el restaurante, los actos cívicos y culturales, las asesorías, las representaciones deportivas y académicas en la comunidad, las convivencias, las salidas de campo, etc. De acuerdo con la Teoría del Reconocimiento, por medio de estas quejas los estudiantes reclaman no solo condiciones de amor, de afecto y de un ambiente cálido en el aula de clase y en la institución educativa, sino solidaridad de los funcionarios educativos para que brinden estas condiciones a las que tienen derecho y se hagan efectivas en la formación y en los procesos de aprendizaje que se orientan; es un reclamo hacia la solidaridad, que solo se ofrece cuando se conocen las situaciones y dolencias del otro. En ocasiones esta falta de interacción conduce a que se origine un distanciamiento entre el docente y el estudiante que puede ser interpretado por este último como indiferencia e invisibilización, y lo conduce a una falta de confianza para aclarar dudas, hacer preguntas, expresarse espontáneamente, etc., lo cual frena el proceso de aprendizaje en el saber de la lengua extranjera. Asimismo, el estudiante reclama del docente cercanía, que no acontezca que "a pesar de contar con tu profesor, te veías sometido a un sistema de educación a distancia, o autodidacta" (Estudiante 3).

También hay quejas por algunas maneras de interacción negativas de los docentes, generadoras de conflictos entre compañeros de clase, que a su vez se relacionan con asuntos como el bullying y la indiferencia frente a situaciones problemáticas que reclaman de su autoridad y de su liderazgo para poner orden o invitar a reflexionar sobre la convivencia y que, al mismo tiempo, ponen límites a la participación libre de los estudiantes:

"[Me quejo] de la indiferencia que muestran algunos docentes hacia los problemas dentro del aula como son las burlas". (Estudiante 18)

"[Me quejo] que el maestro exigiera en todo momento el silencio en el aula". (Estudiante 26)

"Me quejo de docentes que no dejan que los estudiantes se expresen libremente y de controlar hasta su apariencia física y tratar de cambiar su identidad y pensamientos". (Estudiante 18)

En los encuestados se percibe molestia frente a acciones de los docentes como: indiferencia ante problemas entre estudiantes (Estudiante 18), exigencia permanente de silencio en el aula (Estudiante 26); control excesivo sobre la presentación personal, en cuanto a la apariencia física, a cómo se lleva el uniforme, la ropa, el cabello, etc.; cuestionan sobre todo el tono y el momento - el menos adecuado- para decirlo. En términos de la Teoría del Reconocimiento, cada vez que el docente se acerca a un estudiante a exigirle silencio o que lleve su apariencia o presentación personal de una manera diferente y lo hace de manera colectiva, con tono de voz alto y sin respeto en la solicitud, se trata de quejas direccionadas a la afectividad, aspecto que, en la dimensión del amor, el estudiante podría leer como rechazo por parte del docente.

\section{Conclusión}

En el desarrollo de este artículo se expusieron los resultados de un proyecto de investigación en el aula en el que se indagó a los estudiantes de un programa de Licenciatura en lenguas extranjeras, por situaciones en sus clases de inglés, vividas en la escuela, el colegio o en el tiempo que llevan en la universidad, y de las cuales se quejan o manifestaron alguna inconformidad o alguna expectativa, tanto desde las características de la clase de inglés, como de las cualidades que resaltan en el docente de esta área. Dichas quejas y expectativas fueron elaboradas gracias a la implementación de un conjunto de talleres pedagógicos realizados con los estudiantes en el marco de una de las asignaturas del componente pedagógico del plan de estudio.

El análisis que se hizo a los contenidos de estas quejas permitió responder la pregunta de 
Tabla 2. Síntesis segundo núcleo temático.

\begin{tabular}{|c|c|}
\hline Queja & Expectativa \\
\hline $\begin{array}{l}\text { Sobre la falta de profesionalismo de algunos } \\
\text { docentes: un llamado a la atención cognitiva. }\end{array}$ & $\begin{array}{l}\text { Que le guste su materia y manifieste autoridad en ella y en cómo la } \\
\text { enseña. } \\
\text { Que se le note la buena voluntad y la creatividad en sus clases, así } \\
\text { como una actitud dinámica. } \\
\text { Que le exija al estudiante y se interese por su progreso. } \\
\text { Que se esmere en ofrecer unas clases agradables que lleven al } \\
\text { estudiante a aprender y a auto exigirse. }\end{array}$ \\
\hline $\begin{array}{l}\text { Sobre algunos docentes de inglés por su } \\
\text { comportamiento poco cálido: un llamado a } \\
\text { la dedicación emocional. }\end{array}$ & $\begin{array}{l}\text { Que el docente ofrezca al estudiante un trato respetuoso, } \\
\text { considerado, cálido y afectivo. } \\
\text { Que se note y viva su calidad humana. } \\
\text { Que se le note que ser profesor es su vocación profesional. } \\
\text { Que sea dedicado y atento con los estudiantes, que los escuche y } \\
\text { les dedique tiempo. }\end{array}$ \\
\hline $\begin{array}{l}\text { Sobre algunos docentes de inglés que } \\
\text { no interactúan con ellos: un llamado a la } \\
\text { valoración social y a la interacción. }\end{array}$ & $\begin{array}{l}\text { Que brinde un trato amable, paciente y tolerante. } \\
\text { Que escuche a su estudiante y se interese por lo que le acontece. } \\
\text { Que tenga una actitud de acogida, de comprensión. } \\
\text { Que favorezca un ambiente de aprendizaje donde se sientan seguros } \\
\text { y confiados para interactuar con otros en la clase. } \\
\text { Que se acerque al estudiante para conversar con él, incluso en } \\
\text { momentos y espacios por fuera del aula de clase. } \\
\text { Que sea un docente cercano, accesible y no distante e indiferente. } \\
\text { Prudente, humilde y oportuno para llamar la atención a los } \\
\text { estudiantes, preferiblemente de manera individual. }\end{array}$ \\
\hline
\end{tabular}

Fuente: elaboración propia.

investigación que orientó este estudio, tomando como apoyo teórico la pedagogía y la Teoría del Reconocimiento. Gracias a este apoyo se pudo develar que las quejas de los estudiantes giran, de un lado, alrededor de algunas características que se pudieron identificar de la clase de inglés, que no favorecen su proceso de formación y de aprendizaje, relacionadas con los contenidos, el método de la clase, los recursos de apoyo a la didáctica y la evaluación. De otro lado, se refieren a algunas cualidades del docente de esta área que tampoco contribuyen favorablemente a orientar la dirección del proceso de enseñanza y aprendizaje, relacionadas con la falta de profesionalismo, su comportamiento poco cálido y su incapacidad para comunicarse e interactuar positivamente con los estudiantes.
Las quejas en relación con estos aspectos indican, en sí mismas, las expectativas que tienen los estudiantes respecto a sus clases de inglés, así como a los profesores de esta área. Si las expectativas de los estudiantes frente a estos dos temas se concretaran, acercarían la clase a unas características y el rol del docente a unas cualidades más adecuadas y justas, toda vez que favorecerían el reconocimiento del estudiante en términos de amor, derecho y solidaridad.

Por lo que expresan los estudiantes en sus narraciones, se pudo notar que estas quejas revelan la implementación de acciones pedagógicas y didácticas propias de un modelo tradicional, en cuanto a la manera como se generan y se dan ciertas dinámicas de interacción en la clase y en cómo se 
orienta el proceso de enseñanza del inglés. Estas razones pueden explicar en parte las dificultades que se tienen con el aprendizaje de la lengua extranjera, pues con estos procederes tradicionales en la clase, de alguna manera se impide que se logren procesos de formación integral a los estudiantes desde el área, que tengan en cuenta sus posibles necesidades en relación con las dimensiones del amor, el derecho y la solidaridad y que se pueden solventar con prácticas educativas significativas. Por supuesto, ello dificulta los procesos de aprendizaje en la lengua extranjera en términos de lo que puede entenderse como un aprendizaje significativo, y no ofrece a los estudiantes condiciones justas indispensables para su proceso formativo de ciudadanos integrales, tal y como se logra leer en los fines de la educación colombiana.

Desde la Teoría del Reconocimiento (Honneth, 1997), estas características pedagógicas y didácticas de las clases de inglés afectan la dimensión del amor del estudiante, quien espera más calidez en los procesos. Pero no solo la del amor; en sus testimonios dejan ver que los motivos por los cuales se quejan atentan contra ese derecho que tienen por ley a que se comprometan con una educación con calidad. Si la meta es lograr en los estudiantes procesos de formación integral, ellos mismos nos revelan qué hace falta en términos de una enseñanza justa, tal como lo demandan en los que han sido sus reclamos. En ese orden de ideas, se pueden plantear las siguientes implicaciones pedagógicas y didácticas que acerquen la enseñanza del inglés a las expectativas de los estudiantes que necesariamente aluden al docente y a su manera de enseñar:

- La identificación de la necesidad que tienen los estudiantes de ser reconocidos en cuanto a sus requerimientos de amor, derecho y solidaridad y plantear acciones concretas en la enseñanza de este saber específico que contribuya a resolver tales necesidades en las medidas de las posibilidades.

- La selección cuidadosa - y con funcionalidadde contenidos de enseñanza y de aprendizaje, y el diseño de materiales variados y de recursos de apoyo a la didáctica.
- La planeación de métodos, estrategias y actividades para la enseñanza y la evaluación de los contenidos propios del saber de la lengua extranjera, que sea sensible a las necesidades e intereses de los estudiantes, a nivel afectivo y cognitivo; las necesidades e intereses del contexto, a nivel social, cultural, histórico, político y económico; la naturaleza social y humana del saber de la lengua extranjera; así como los estilos cognitivos de los estudiantes.

No se puede dejar de mencionar lo que todo esto implica para el docente de la lengua extranjera, en cuanto a: su permanente capacitación y actualización en lo que va aconteciendo en este saber desde la investigación; la reflexión personal de cómo se siente en el ejercicio de la docencia y de la calidad con que está desarrollando su rol profesional; su actitud con los estudiantes y de lo que puede hacer para mejorar o fortalecer unas relaciones para lograr mayor calidad, tal cual las reclaman en los testimonios.

Finalmente, y a manera de recomendación, sería interesante explorar a futuro, con fundamento en lo aportado por esta investigación, los dos siguientes temas: uno relacionado con los estudiantes, explorar aspectos positivos del aprendizaje de la lengua extranjera y qué, en general, los hace sentir motivados para aprender el inglés. En segundo lugar, indagar las quejas por parte de los docentes de la lengua extranjera, explorando reclamos, demandas o denuncias que, al ser tenidas en cuenta, contribuirían a la cualificación de los procesos de enseñanza que orientan en sus estudiantes.

Queda suficiente motivación para mejorar la acción social y educativa justa como docentes, específicamente de la lengua extranjera y para que ellos también expresen sus quejas y reclamos. Mediante este ejercicio, se ayudaría a enfrentar de mejor manera en el desempeño de su rol como maestros e investigadores de los procesos de enseñanza y aprendizaje, ampliando la mirada del docente frente al currículo oculto y como intelectual de la pedagogía que lo moviliza a implementar reflexiones y transformaciones en pro de ofrecer a sus estudiantes experiencias de enseñanza formativas y justas. 


\section{Referencias}

Álvarez, C. y González, E. (1998). Lecciones de didáctica general. Colombia: Edinalco Ltda.

Baggini, J. (2012). Ethics. The Big Questions. [eBook]. UK: Quercus.

Benjumea, M., Gutiérrez, A., Jaramillo, O., Mesa, A., y Pimienta, A. (2011). Formación ciudadana (FC) y educación para la ciudadanía (EpC). Aproximaciones conceptuales y mínimos compartidos. Revista Temas, 5, 211-224. https://doi.org/10.15332/ rt.v0i5.695

Bustelo, M. y Lombardo, E. (2007). Políticas de igualdad en España y en Europa. Madrid: Cátedra. https://doi. org/10.5944/empiria.15.2008.1205

Congreso de la República de Colombia. (2012). Ley 115 de febrero 8 de 1994. Ley General de Educación. Bogotá: Editorial Unión Ltda.

Connelly, F. y Clandinin, D. (1995). Relatos de experiencia e investigación narrativa. Déjame que te cuente. En Larrosa (Eds.). Déjame que te cuente. Ensayos sobre narrativa y educación, (pp.11-59). Barcelona: Laertes.

Constitución Política de Colombia (1991). Bogotá D.C.: Panamericana Editorial.

Díaz, A. y Quiroz, R. (2005). Educación, instrucción y desarrollo. Medellín: Imprenta Universidad de Antioquia. XVIII.

Echavarría, C. (2011). Concepciones de ciudadanía y de ejercicio ciudadano en un grupo de jóvenes en situaciones de protección. Revista Educación y Pedagogía, 23(59), 197-211. https://doi. org/10.15332/rt.v0i5.693

Egg, A. (1999). El taller: una alternativa de renovación pedagógica. Río de la Plata: Editorial Magisterio.

Galeano, M. E. (2004). Diseño de proyectos en la investigación cualitativa. Medellín: Fondo Editorial Universidad EAFIT.
Ghiso, A. M. y Ospina, V. Y. (2010). Intimidación y maltrato entre escolares. Revista Conversaciones Pedagógicas, 35-49, Universidad Católica de Oriente. N/A fasc. N/A (pp.).

Honneth, A. (1997). La lucha por el reconocimiento: Por una gramática moral de los conflictos sociales. Barcelona: Editorial Crítica.

Mateos, P. H. (2015). Narrativas de la violencia: las voces infanto-adolescentes como parrhesia. [Libro digital, PDF]. Ciudad Autónoma de Buenos Aires: CLACSO.

Mejía, S. (2017). Formación ciudadana y justicia social en la educación desde la teoría del reconocimiento: discursos sobres las prácticas de los líderes del proyecto Colegios Maestros de Medellín. Tesis de doctorado Facultad de Educación de la Universidad de Antioquia. https://doi.org/10.14422/pym. v0i356.3070

Orrego, L. y Díaz, A. (2010). Empleo de estrategias de aprendizaje de lenguas extranjeras: inglés y francés. Revista Íkala, 15(24), 108.

Orozco, L. (2002). La formación integral como base para definir estrategias de un pensamiento lúcido y pertinente. Revista Debates, 32, 26-38.

Ramírez, N. (2014). "Narrativas de vida y Memorias". Conflicto escolar en el Colegio Santa Librada. Revista Latinoamericana de Ciencias Sociales, Niñez y Juventud, 12(1), 201-210. https://doi. org/10.11600/1692715x.12111042613

Rojas, A. (2009, enero-abril). Didáctica Crítica, critica la crítica Educación Bancaria. Integra Educativa No 4, II (1), 93-108.

Salm, R. y Gómez, E. (2000). Un estudio de conflictos estudiantiles en Colombia. Investigación Educativa y Formación Docente, 5/6, 179-193.

Tobón, S. (2010). Formación integral y competencias. Pensamiento complejo, currículo, didáctica y evaluación. Centro de Investigación en Formación y Evaluación (CIFE), Bogotá D.C.: Ecoe Ediciones.

Van Dijk, T. (2003). El discurso como estructura y proceso. Barcelona: Editorial Gedisa. 